\title{
Editorial: Innovation und Forschung in der Arbeits(zeit)organisation
}

\author{
Anna ARLINGHAUS, Johannes GÄRTNER, Sylvia RABSTEIN, Sebastian SCHIEF, Anne Marit WÖHRMANN ${ }^{1}$
}

Liebe Leserinnen und Leser,

als wir im Oktober des Jahres 2019 unser jährliches Symposium der Arbeitszeitgesellschaft zum Thema Innovation in Forschung und Praxis in Wien durchführten, haben wir noch keine Sekunde daran gedacht, dass eine Pandemie auf uns zukommen würde. Wie die meisten anderen auch wurden wir im weiteren Verlauf von den Entwicklungen regelrecht überrollt. Dies hat selbstverständlich die Leben der allermeisten von uns gründlich durcheinandergewirbelt, und auch die Art und Weise, wie wir arbeiten, hat sich fundamental verändert. Viele von uns arbeiten nun von zu Hause, Meetings finden häufig online statt. Grosse Unternehmen wie Siemens denken inzwischen darüber nach, einen grossen Teil der MitarbeiterInnen generell von $\mathrm{Zu}$ hause aus arbeiten zu lassen (vgl. dpa-infocom 2020). Als wir also im Oktober über Innovationen in der Arbeitszeitorganisation und der Arbeitsorganisation sprachen, hatten wir noch nicht die geringste Ahnung, dass eine Pandemie wie die Corona-Krise wie ein Katalysator wirken und unsere Art zu arbeiten radikal verändern würde. Noch wissen wir natürlich nicht, wie nachhaltig die Veränderungen in der Arbeits(zeit)organisation sein werden, aber viele Indizien sprechen dafür, dass wir uns in einer Zeitenwende befinden.

Naturgemäss konnte unser Symposium und damit auch unser Sonderheft noch nicht aufzeigen, welche Entwicklungen durch die Pandemie entstanden oder verstärkt wurden, aber wir können zeigen, welche Innovationen schon vorhanden waren, als diese Krise ihren Anfang nahm. In diesem Sonderheft werden Artikel zum Thema Schichtarbeit, soziale Teilhabe und atypische Arbeitszeiten sowie Überstunden und Work-Life-Balance besprochen. Den Anfang macht ein Beitrag von Anne Marit Wöhrmann, Grit Müller und Kathrin Ewert. In ihrem Beitrag Shift Work and Work-Family Conflict: A Systematic Review geben die Autorinnen einen systematischen Überblick über den Forschungsstand zum Thema der Rolle von Schichtarbeit

\footnotetext{
${ }^{1}$ Dr. Anna Arlinghaus ist Arbeitspsychologin und arbeitet als Beraterin bei XIMES GmbH. Sie beschäftigt sich mit der ergonomischen Gestaltung von Arbeitszeiten: arlinghaus@ximes.com. PD Dr. Johannes Gärtner ist Gründer und Geschäftsführer der XIMES GmbH. Neben seiner Beratungstätigkeit bei der XIMES GmbH unterrichtet Dr. Gärtner im Fach Wirtschaftsinformatik an der Technischen Universität Wien: gaertner@ximes.com. Dr. Sylvia Rabstein ist Statistikerin und Epidemiologin und arbeitet am Kompetenz-Zentrum Epidemiologie des Instituts für Prävention und Arbeitsmedizin der Deutschen Gesetzlichen Unfallversicherung und Institut der Ruhr-Universität Bochum (IPA): rabstein@ipa-dguv.de. Dr. Sebastian Schief ist Soziologie und arbeitet im Bereich Soziologie, Sozialpolitik, Sozialarbeit der Universität Fribourg/Schweiz: sebastian.schief@unifr.ch. Dr. Anne M. Wöhrmann ist Psychologin und forscht zu Arbeitszeitgestaltung, Work-Life Balance und Gesundheit bei der Bundesanstalt für Arbeitsschutz und Arbeitsmedizin (BAuA, Dortmund): woehrmann.annemarit@baua.bund.de.
} 
für den Konflikt zwischen Familie und Erwerbsarbeit. Die Autorinnen führen dabei eine systematische Literaturrecherche in PubMed und EBSCO durch, um Studien zu diesem Thema in den Jahren 1990 bis 2017 zu identifizieren. Insgesamt nehmen sie 36 Artikel auf, die den Suchkriterien entsprechen. Die Ergebnisse zeigen, dass SchichtarbeiterInnen einen stärkeren Konflikt zwischen Familie und Erwerbsarbeit erleben als ArbeiterInnen mit normalen täglichen Arbeitszeiten. Innerhalb der verschiedenen Studien wurden diverse Schichttypen und -charakteristika untersucht. Die Resultate deuten auf einen stärkeren Konflikt zwischen Familie und Erwerbsarbeit, insbesondere bei ArbeiterInnen in Nachtschicht und in Schichten mit Wochenendarbeit, hin.

Maren Evers untersucht in ihrem Beitrag Neue Gestaltungsoptionen für humane Nacht- und Schichtarbeit? - Experteneinschätzungen am Beispiel Digitalisierung die Möglichkeiten der Verbesserung der Organisation von Schichtarbeit mit den Mitteln der Digitalisierung. Im Zuge der Debatte um Industrie 4.0 und Digitalisierung wurden von Seiten der Produktionstechnik erhebliche Versprechungen hinsichtlich einer Verbesserung von Arbeitsqualität gemacht. Auch für die Gestaltung von Nacht- und Schichtarbeit liegen Konzepte, wie z. B. ein Schicht-Doodle, vor. Der Beitrag fragt danach, inwiefern sich durch Digitalisierung wirklich Chancen für eine humane Gestaltung von Nacht- und Schichtarbeit ergeben. Ergebnisse aus 40 ExpertInneninterviews lassen darauf schliessen, dass im Einsatz neuer Technologien kein hinreichender Lösungsansatz zu sehen ist. Problemlagen wie Management-Defizite in der Gestaltung betrieblicher Abläufe und Fehlanreize durch monetären Belastungsausgleich bleiben grundlegende Hemmnisse, die es zu überwinden gilt.

Bettina Stadler, Annika Schönauer, Anna Arlinghaus, Bernhard Saupe und Hubert Eichmann diskutieren in ihrem Beitrag Das Leben ist Synchronisation - Möglichkeiten und Formen sozialer Teilhabe vor dem Hintergrund atypischer Arbeitszeiten inwiefern sogenannte atypische Arbeitszeiten, also Schichtarbeit, lange Arbeitszeiten, Wochenendarbeit und flexible Arbeitszeiten, die Möglichkeiten, am sozialen Leben teilzunehmen, beeinflussen. Der Beitrag präsentiert Ergebnisse einer qualitativen Erhebung zu arbeitszeitbedingten Beeinträchtigungen sozialer Teilhabe in Österreich. Der Fokus liegt dabei auf langen Arbeitszeiten, Nachtarbeit und der Planbarkeit von Arbeitszeit in den Bereichen Pflege, Sicherheitsdienstleistung, Hotellerie und IT-Projektarbeit. Soziale Teilhabe wird insbesondere durch atypische Lagen von Arbeitszeit und fremdgesteuerte Flexibilität beeinträchtigt. Die Probleme der Synchronisation unterschiedlicher Lebensbereiche potenzieren sich, wenn lange Arbeitszeiten und geringe Mitgestaltungsmöglichkeiten hinzukommen. Mit zunehmender Belastung sind am stärksten regelmässige Termine gefährdet, gefolgt von Sozialkontakten ausserhalb der Familie. Schliesslich bleiben nur Kontakte innerhalb der Kernfamilie erhalten.

Corinna Brauner, Anne Marit Wöhrmann, Nils Backhaus, Frank Brenscheidt und Anita Tisch diskutieren in ihrem Beitrag Überstunden, Ausgleichsmöglichkeiten, Gesundheit und Work-Life Balance repräsentative Ergebnisse der BAuA-Arbeitszeitbefragung des Jahres 2017. Sie können anhand repräsentativer Daten von 7765 Befragten der BAuA-Arbeitszeitbefragung 2017 zeigen, dass Überstunden in Deutschland weit verbreitet sind. Beschäftigte arbeiten im Durchschnitt 3,9 Stunden pro Woche länger als vertraglich vereinbart, wobei sich Unterschiede nach Geschlecht, Vollzeittätigkeit, Qualifizierung und Berufen zeigen. Über die Hälfte sind 
transitorische Überstunden, die durch Freizeit ausgeglichen werden. Ein Viertel wird ausbezahlt und jede fünfte Überstunde wird nicht abgegolten. Regressionsanalysen deuten auf einen negativen Zusammenhang von Überstunden mit Gesundheit und Work-Life-Balance hin. Dies gilt sowohl für transitorische Überstunden als auch bei Überstunden ohne Freizeitausgleich. Stratifizierte Analysen zeigen teilweise abweichende Muster für Beschäftigte in Nacht- und Schichtarbeit sowie bei Beschäftigten, die Überstunden hauptsächlich aus privaten Gründen machen.

Anna Arlinghaus und Johannes Gärtner stellen in ihrem Forumsbeitrag Unfallrisiko verschiedener Schichtsysteme - Simulation mit dem XIMES-Risikorechner eine technologische Möglichkeit der Kalkulation von Unfallrisiken in der Schichtarbeit vor. Die AutorInnen gehen - aufbauend auf eine große Meta-Studie und einem umfangreichen Korpus an Forschungsarbeiten zum Thema - davon aus, dass Arbeitszeiten das Fehler- und Unfallrisiko der Beschäftigten beeinflussen können. Um die Risiken verschiedener Arbeitszeit- bzw. Schichtmodelle zu bewerten, wurden fünf häufig anzutreffende Standardmodelle mit dem XIMES-Risikorechner analysiert und verglichen. Inkludiert wurden kurz vorwärts und lang rückwärts rotierte Schichtpläne, 12-Stunden-Modelle und lange Tagarbeit. Die Ergebnisse zeigen bei allen untersuchten Systemen erhöhte Risiken im Vergleich zu Tagarbeit mit 8-Stunden-Schichten, jedoch treten sowohl im Gesamtrisiko als auch in den Spitzen an einzelnen Arbeitstagen grosse Unterschiede auf. Das kurz vorwärts rotierte System schneidet dabei auf beiden Dimensionen am besten ab. Die AutorInnen hoffen, dass der Risikorechner und die Ergebnisse für die Evaluierung und präventive Arbeitszeitgestaltung genutzt werden können.

Das nunmehr 7. Symposium der Arbeitszeitgesellschaft lieferte spannende Einsichten über innovative Entwicklungen und spannende Forschung. Wir hoffen mit den präsentierten Ergebnissen wichtige Anstösse für die Weiterentwicklung der Arbeits(zeit)organisation in Forschung und Praxis geben zu können. Dies gilt insbesondere und gerade in diesen aussergewöhnlichen Zeiten mit ihren schnellen und grundlegenden Veränderungen. Die Arbeitszeitgesellschaft hat inzwischen ein 8. Arbeitszeitsymposium durchgeführt, das in diesem Jahr aufgrund der Pandemie online abgehalten wurde. Wir hoffen, Sie bald wieder auf einem unserer Symposien persönlich begrüssen zu können!

Eine spannende Lektüre wünscht Ihnen der Vorstand der Arbeitszeitgesellschaft e.V.:

Anna Arlinghaus, Johannes Gärtner, Sylvia Rabstein, Sebastian Schief, Anne Marit Wöhrmann

\section{Quellen}

dpa-infocom (2020). Siemens macht Homeoffice für 140.000 Mitarbeiter möglich. Süddeutsche Zeitung 16.07.2020. Zugriff auf https://www.sueddeutsche.de/karriere/arbeit-siemens-macht-homeofficefuer-140-000-mitarbeiter-moeglich-dpa.urn-newsml-dpa-com-20090101-200716-99-809129 am 16. 07. 2020. 